\title{
INSATISFAÇÃO CORPORAL E COMPORTAMENTO ALIMENTAR EM ATLETAS DE ESPORTES ESTÉTICOS: UMA REVISÃO ${ }^{1}$
}

\author{
Clara Mockdece Neves \\ Universidade Federal de Juiz de Fora, Minas Gerais, Juiz de Fora, Brasil \\ Juliana Fernandes Filgueiras Meireles \\ Universidade Federal de Juiz de Fora, Minas Gerais, Juiz de Fora, Brasil \\ Pedro Henrique Berbert de Carvalho \\ Universidade Federal de Juiz de Fora, Minas Gerais, Governador Valadares, Brasil \\ Maria Elisa Caputo Ferreira \\ Universidade Federal de Juiz de Fora, Minas Gerais, Juiz de Fora, Brasil
}

\begin{abstract}
Resumo
O objetivo foi analisar a produção científica sobre insatisfação corporal e comportamentos de risco para transtornos alimentares em adolescentes atletas de esportes estéticos. Foram analisados 45 estudos publicados entre 2008 e 2015. No Brasil, foram identificadas prevalências semelhantes de insatisfação corporal e comportamento de risco para transtorno alimentar em atletas, possivelmente pela utilização dos mesmos instrumentos de avaliação. Internacionalmente, identificou-se maior variedade de instrumentos avaliativos, o que repercutiu nas diferenças das prevalências desses comportamentos. São incentivados estudos brasileiros que utilizem métodos longitudinais e com grupo controle.
\end{abstract}

Palavras-chave: Imagem Corporal. Comportamento Alimentar. Transtornos da Alimentação. Adolescente.

\section{Introdução}

Os transtornos alimentares são considerados síndromes psiquiátricas, caracterizadas por padrão de comportamento alimentar inadequado e controle patológico da massa corporal (ANDERSON; PETRIE; NEUMANN, 2011). Uma vivência conturbada com o corpo é destacada como um dos critérios diagnósticos para os transtornos alimentares (APA, 2014). A imagem corporal pode ser entendida como a representação mental do corpo (FERREIRA; CASTRO; MORGADO, 2014). Um de seus componentes é a insatisfação corporal, a qual se refere à avaliação negativa do próprio corpo, e é frequente em pacientes com transtorno alimentar (FERREIRA; CASTRO; MORGADO, 2014).

\footnotetext{
${ }^{1}$ Apoio financeiro: Bolsa de mestrado (monitoria UFJF) concedida à primeira autora.
} 
A avaliação da insatisfação corporal e dos comportamentos de risco para transtornos alimentares em atletas tem despertado o interesse de pesquisadores pela possível relação que essas variáveis podem ter com o treinamento de alto rendimento (FORTES, ALMEIDA, FERREIRA, 2012a; FORTES, ALMEIDA, FERREIRA, 2012b; FORTES, FERREIRA, 2011; VIEIRA et al., 2009). Algumas modalidades esportivas são apontadas como desencadeadoras da insatisfação corporal e também como de maior risco para o desenvolvimento de os transtornos alimentares, dentre elas destacam-se aquelas com divisão por classe de peso corporal (por exemplo, tae-kwon-do, judô, jiu-jitsu) e as de caráter estético (ginásticas artística, rítmica e de trampolim, a patinação artística, o nado sincronizado e o ballet clássico) (FORTES, FERREIRA, 2011; FRANCISCO, ALARCÃO, NARCISO, 2012; KLINKOWSKI et al., 2008; PETRIE, GREENLEAF, 2011; VIEIRA et al., 2009).

Nas modalidades estéticas, a aparência física dos atletas pode influenciar na avaliação dos jurados (PETRIE; GREENLEAF, 2011). Em se tratando de adolescentes, as mudanças corporais típicas dessa fase (CONTI, 2008), somada à inserção precoce em programas de treinamento de alto rendimento (MALINA et al., 2013), podem ter sérias consequências na imagem corporal desses jovens (FORTES; ALMEIDA; FERREIRA, 2014a). Dessa forma, esses jovens atletas podem estar mais propensos à insatisfação corporal, e adoção de comportamentos de risco para transtornos alimentares buscando atingir o padrão de corpo ideal para o esporte (ANDERSON, PETRIE, NEUMANN, 2011; FORTES, NEVES et al., 2013; FORTES, OLIVEIRA, FERREIRA, 2012).

Diante da relação que o treinamento de alto rendimento pode apresentar com os comportamentos de risco para transtornos alimentares e a imagem corporal dos atletas, especialmente aqueles praticantes de esportes de caráter estético, ainda existe uma lacuna do conhecimento no sentido de analisar estudos que abordem o tema. Sendo assim, o objetivo do presente estudo é analisar a produção científica nacional e internacional referente à avaliação da insatisfação com a imagem corporal e dos comportamentos de risco para transtornos alimentares em adolescentes atletas de modalidades esportivas estéticas.

\section{Métodos}

Realizou-se uma revisão sistemática da literatura por meio de uma busca eletrônica de artigos indexados em quatro bases de dados. Optou-se pela utilização das bases: Scopus, devido ao seu aspecto multidisciplinar; PubMed, por ser considerada uma base de grande relevância na área da saúde; PsycINFO, por ser uma base de referência nas publicações em Psicologia; e Biblioteca Virtual em Saúde (BVS), por ser considerada um base de relevância nacional.

Em relação ao delineamento temporal, estudo de revisão realizado por Ferreira, Castro e Morgado (2014) demonstrou que houve um aumento significativo dos estudos em imagem corporal a partir do ano de 2008. Dessa forma, utilizou-se esse recorte que possibilitará uma revisão mais atualizada sobre o tema. As buscas foram realizadas durante o mês de junho de 2015 .

Visando a alcançar um número representativo da produção científica, optou-se pela utilização do cruzamento do termo "athletes", por meio do operador boleano AND, com as palavras-chave "body image" e "eating disorders", que são termos indexados nos Descritores de Ciências em Saúde (Decs) e no Medical Subject Headings (MeSH) do PubMed. Ademais, utilizou-se também os termos "body dissatisfaction" e "eating behavior" por apresentarem 
relação com o tema de estudo. Em todas as bases de dados, foram usados termos apenas em inglês, pois foi encontrado maior número de achados nessa língua.

$\mathrm{Na}$ Scopus, as palavras-chave foram inseridas no campo de busca com a opção de busca em "article title, abstract, keywords". Ademais, limitou-se a busca por intervalo de datas de 2008 a 2015 e na seção "document type", optou-se por incluir apenas artigos. Na PubMed, selecionou-se a opção "advanced" em "all fields" para possibilitar o cruzamento dos termos. Quanto aos filtros adicionais, optou-se por limitar a sessão "article types" apenas por "journal articles", e em "publication dates" customizou-se o intervalo para de 01 de janeiro de 2008 até o dia no qual a busca foi realizada. Na PsycINFO, realizou "advanced search", inserindo os termos em "any field". Selecionou-se a busca apenas em "journal article" quanto ao tipo de documento e no período de 2008 até 2015. Na BVS, realizou-se uma busca avançada selecionando "todos os índices", o "método integrado" e "todas as fontes" (MEDLINE, LILACS, CENTRAL - Registros de Ensaios Clínicos Controlados, IBECS (Espanha), Index Psi (Psicologia). Seguindo os mesmos critérios anteriores, restringiu-se apenas "artigos" quanto ao "tipo de documento" e quanto ao "ano de publicação", apenas de 2008 a 2015.

Após a inclusão dos estudos encontrados seguindo os critérios acima, eles foram selecionados por dois pesquisadores independentes. Foram traçados como critérios de exclusão: a) estudos em duplicata pelas diferentes bases de dados; b) que não apresentaram resumo disponível na base de dados; c) estudos de metodologia não empírica (como revisões ou artigos de diretrizes); d) publicados em outros idiomas que não o inglês, o espanhol ou o português; e) de construção e/ou validação de escalas; f) estudos que não incluíam modalidades estéticas (ginástica artística, ginástica rítmica e de trampolim, patinação artística, nado sincronizado ou ballet clássico (PETRIE, GREENLEAF, 2011; FORTES; ALMEIDA; FERREIRA, 2014a); g) com população infantil (abaixo de 10 anos - ONIS et al., 2007) e adulta (acima de 21 anos - ONIS et al., 2007). Por fim, buscou-se a versão na íntegra dos artigos incluídos com o auxílio das próprias bases e do Portal de Periódicos Capes, e foram excluídos aqueles que não foram encontrados na íntegra.

Após a aplicação dos critérios de inclusão e exclusão, foi realizada análise dos artigos na íntegra, a qual possibilitou extrair as seguintes informações: 1) autor e ano; 2) país de publicação; 3) característica da amostra; 4) modalidades esportivas; 5) método utilizado; e 6) instrumentos avaliativos utilizados; 7) áreas de estudos de onde provêm os artigos analisados. Além disso, a análise e a interpretação dos resultados encontrados em cada pesquisa foram realizadas concomitantemente com a organização e discussão. Optou-se por analisar separadamente as publicações nacionais e internacionais.

\section{Resultados}

A Tabela 1 apresenta, no primeiro momento, o número de artigos identificados em cada base de dados a partir das combinações de palavras-chave e, no segundo momento, os critérios de exclusão para a seleção dos estudos. A Figura 1 apresenta a descrição do processo de exclusão, considerando o total de artigos identificados em todas as bases de dados. 
Tabela 1 - Número de publicações encontradas a partir de cada cruzamento das palavraschave e excluídas segundo os critérios adotados.

\begin{tabular}{lcccc}
\hline & SCOPUS & PUBMED & BVS & PsycINFO \\
\hline $\begin{array}{l}\text { "body image" AND "athletes" } \\
\text { "body dissatisfaction" }\end{array}$ AND & 50 & 152 & 105 & 79 \\
"athletes" & & 26 & 30 & 31 \\
"eating disorders" AND "athletes" & 199 & 161 & 148 & 115 \\
"eating behavior" AND "athletes" & 60 & 144 & 67 & 53 \\
\hline Total inicial & $\mathbf{4 6 1}$ & $\mathbf{4 3 7}$ & $\mathbf{3 5 0}$ & $\mathbf{2 7 8}$ \\
\hline Critérios de exclusão & & & & \\
\hline Etapa 1 - Artigos em duplicata & 230 & 269 & 244 & 147 \\
Etapa 2 - Resumos não disponíveis & 2 & 3 & 0 & 3 \\
Etapa 3 - Estudos não empíricos & 33 & 42 & 3 & 31 \\
$\begin{array}{l}\text { Etapa 4 - Idioma } \\
\text { Etapa 5 - Estudos de validação }\end{array}$ & 23 & 15 & 10 & 19 \\
Etapa 6- Esportes não estéticos & 82 & 3 & 8 & 7 \\
Etapa 7 - População infantil e & 37 & 17 & 73 & 46 \\
adulta & & & & 15 \\
Etapa 8 - Não disponível na & 9 & 2 & 0 & 6 \\
integra & & & & \\
Total para análise & $\mathbf{3 6}$ & $\mathbf{3}$ & $\mathbf{2}$ & $\mathbf{4}$ \\
\hline
\end{tabular}




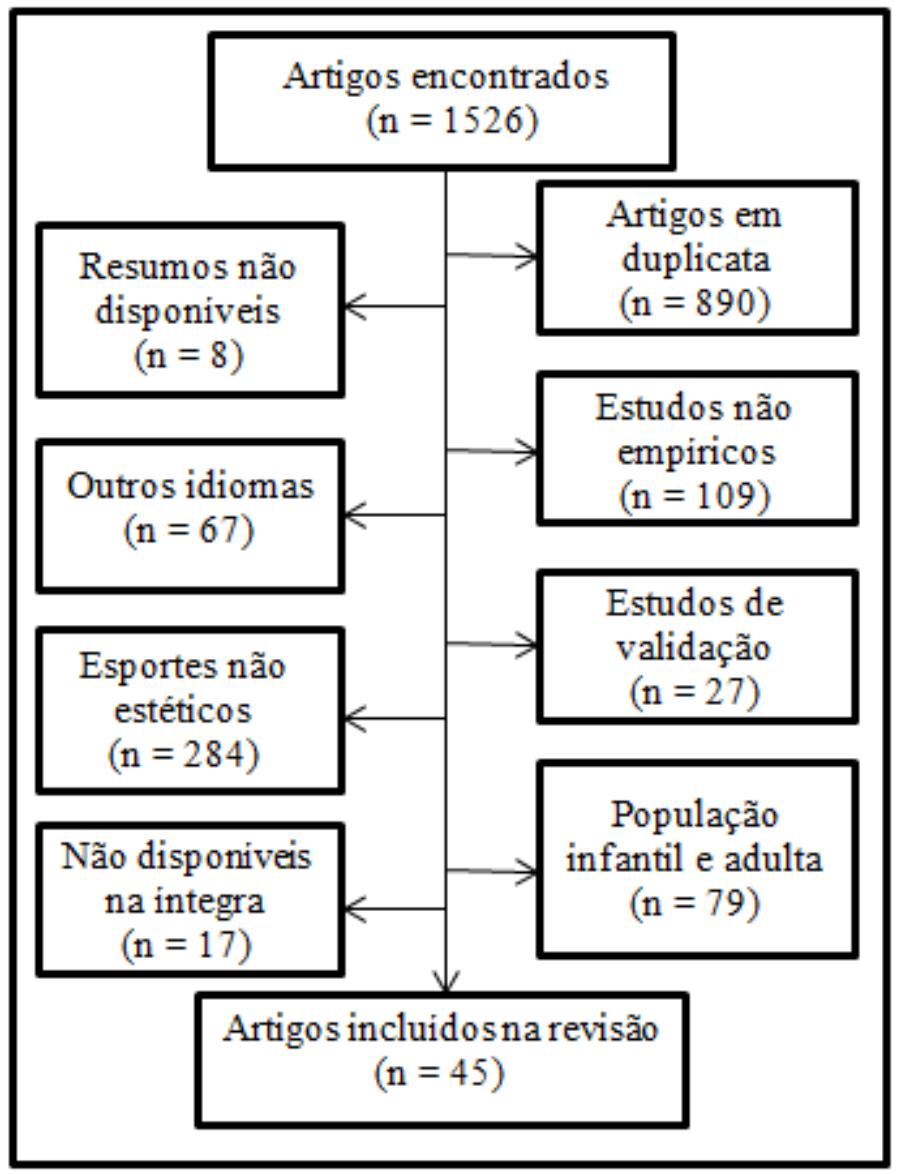

Figura 1. Número de publicações excluídas considerando o total de artigos.

\section{Discussão}

A insatisfação corporal, bem como os comportamentos de risco para transtornos alimentares em atletas, deve ser alvo de atenção por parte dos profissionais que lidam diretamente com esse público, tendo em vista a manutenção da saúde desses. Dessa forma, a presente revisão sistemática teve como objetivo analisar a produção científica nacional e internacional referente à avaliação da imagem corporal e dos comportamentos de risco para transtornos alimentares em adolescentes atletas de modalidades esportivas estéticas.

\section{As pesquisas brasileiras sobre imagem corporal e comportamentos de risco para transtornos alimentares}

Em se tratando de estudos que incluem esportes estéticos, o Brasil tem posição de destaque na literatura, por ser o país que apresenta maior quantidade de pesquisas realizadas. Além disso, salienta-se que esses estudos apresentam algumas características em comum. Inicialmente, quanto aos métodos empregados, os 16 estudos brasileiros incluídos na presente revisão são transversais e quantitativos. O delineamento transversal apresenta uma limitação intrínseca à sua característica: a impossibilidade de estabelecer relação de causa e efeito. Como não foram identificados estudos longitudinais realizados no Brasil, ainda são 
recomendados estudos que busquem preencher essa lacuna de conhecimento.

Ademais, as pesquisas nacionais $(\mathrm{n}=16)$ valeram-se do Eating Attitudes Test-26 para avaliação dos comportamentos de risco para transtornos alimentares. A prevalência de adolescentes que foram considerados com esses comportamentos, de acordo com o ponto de corte estabelecido pelo instrumento (EAT $>20)$, manteve-se semelhante nos estudos identificados. Como exemplo, é possível citar o estudo realizado por Fortes, Kakeshita et al. (2013), o qual comparou a presença desses comportamentos em atletas e não atletas de ambos os sexos. Entre as meninas, $18,1 \%$ das atletas e $26,1 \%$ das não atletas obtiveram pontuação maior que 20 no EAT-26. Já entre os meninos, 14,4\% e 15,3\% dos atletas e não atletas, respectivamente, apresentaram comportamentos de risco para transtornos alimentares. Segundo este estudo, o grupo de não atletas apresentou maior descontentamento com o próprio corpo quando comparados aos atletas.

Com relação à insatisfação corporal, as pesquisas nacionais que avaliaram esse construto ( $\mathrm{n}=13)$ utilizaram o mesmo instrumento: Body Shape Questionnaire. A versão para adolescentes deste instrumento estabelece quatro níveis de insatisfação com o corpo (livre; leve; moderada; e grave insatisfação). Porém, os estudos identificados agruparam os três níveis com algum grau de insatisfação (leve; moderada; e grave insatisfação) para "indivíduos insatisfeitos". Na maioria dos estudos, a prevalência de insatisfação com o corpo foi maior em meninas do que meninos, o que pode ser exemplificado pelos achados de Fortes, Conti e Ferreira (2012), que encontraram 38,8\% das meninas e 14,7\% dos meninos insatisfeitos com seu corpo. Ressalta-se que esse padrão (meninas mais insatisfeitas do que meninos) é o mesmo observado na população geral (FERREIRA; CASTRO; MORGADO, 2014). Entretanto, como o BSQ avalia as preocupações com a forma e peso corporal direcionado à magreza, é possível que os meninos não sejam bem representados por esse instrumento, uma vez que sua preocupação principal é, na maioria das vezes, voltada para a muscularidade (FERREIRA; CASTRO; MORGADO, 2014).

É necessário evidenciar que os instrumentos em questão (Eating Attitudes Test-26 e Body Shape Questionnaire), apesar de serem os mais utilizados, não foram desenvolvidos para amostras específicas de atletas. Todavia, parece que mantiveram boas qualidades psicométricas para as amostras dos estudos (alpha de Cronbach acima de 0,70 - STREINER, 2003). Sendo assim, a escolha desses instrumentos para avaliação de atletas no Brasil pode ser justificada. Afinal, para a avaliação de seus construtos, são os instrumentos disponíveis no país.

Apesar das semelhanças destacadas acima, também é necessário ressaltar particularidades de alguns estudos. O estudo de Fortes e Ferreira (2011) merece destaque pela tentativa de comparar a insatisfação corporal e os comportamentos de risco para transtornos alimentares entre atletas adolescentes de diversas modalidades esportivas e diferentes níveis competitivos. Os autores encontraram que, no geral, essas variáveis foram semelhantes em esportistas adolescentes, praticantes de modalidades com características distintas. Foram identificadas diferenças em poucas modalidades esportivas, entre elas o nado sincronizado. Isso reforça a necessidade de estudos em populações específicas.

Embora a maioria das pesquisas brasileiras identificadas na presente revisão tenha avaliado diversas modalidades esportivas em conjunto, cinco estudos nacionais buscaram verificar as particularidades de algumas modalidades, avaliando-as de maneira específica. Perini et al. (2009) e Vieira et al. (2009) buscaram verificar a presença de comportamentos de risco para transtornos alimentares e de insatisfação corporal em atletas de elite do nado 
sincronizado e ginástica rítmica, respectivamente. Ambos os autores avaliaram tanto um grupo de atletas quanto um grupo de adolescentes não atletas (controle). Além disso, dentro da categoria de atletas, as participantes foram divididas por faixa etária - júnior e sênior no caso de Perini et al. (2009) e de 10 a 14 anos e acima de 15 anos no caso de Vieira et al. (2009). Considerando as particularidades de seus esportes, ambos os estudos identificaram os grupos mais jovens - juniores (PERINI et al., 2009) e 10 a 14 anos (VIEIRA et al., 2009) como os que apresentaram maiores níveis de insatisfação corporal e de comportamentos de risco para transtornos alimentares. Isso ressalta a necessidade de um acompanhamento multidisciplinar durante o processo de treinamento de alto rendimento em idades precoces.

Somado a esses, três outros estudos brasileiros avaliaram os comportamentos de risco para transtornos alimentares em atletas de ginástica artística. As pesquisas buscaram associar fatores psicológicos - ansiedade (FORTES; ALMEIDA; FERREIRA, 2013a); perfeccionismo e estado de humor (NEVES et al., 2013); e influência da mídia (FORTES; ALMEIDA; FERREIRA, 2014b) - a esses comportamentos. Os autores apontam a necessidade de incentivo a programas de intervenção tendo em vista a saúde psicológica de atletas, bem como a realização de mais pesquisas nessa modalidade que busquem avaliar os comportamentos de risco para transtornos alimentares e possíveis variáveis influenciadoras.

Nesse mesmo sentido, foram identificados estudos que relacionaram os comportamentos de risco para transtornos alimentares a outras variáveis avaliando diversas modalidades esportivas em conjunto. Os estudos de Fortes e Ferreira (2013) e de Fortes, Neves et al. (2013), por exemplo, apresentam características semelhantes pela tentativa de relacioná-los ao comprometimento psicológico ao exercício. Fortes e Ferreira (2013) identificaram que a restrição alimentar patológica (dieta) foi mais frequente em atletas com elevado grau de comprometimento psicológico ao exercício para ambos os sexos, o que não diferiu nos demais atributos do comportamento alimentar (bulimia e autocontrole oral). Corroborando com esses achados, Fortes, Neves et al. (2013) verificaram que a insatisfação corporal e o comprometimento psicológico ao exercício foram fatores predisponentes para o comportamento alimentar de risco em atletas de esportes estéticos. Dessa forma, parece que o sentimento de elevado comprometimento ao esporte influencia os comportamentos de risco para transtornos alimentares de atletas, pois eles tendem a adotar tais condutas a fim de melhorar a própria performance esportiva.

O processo maturacional também foi uma variável associada aos comportamentos de risco para transtornos alimentares e à insatisfação corporal (FORTES, ALMEIDA, FERREIRA, 2012b; FORTES, CONTI, FERREIRA, 2012). Os autores avaliaram tanto a maturação somática quanto a maturação sexual dos atletas. Ambos os estudos concluíram que o processo maturacional exerceu pouca influência sobre os comportamentos de risco para transtornos alimentares, e essa relação foi observada apenas nos atletas do sexo masculino (FORTES, ALMEIDA, FERREIRA, 2012b; FORTES, CONTI, FERREIRA, 2012). Já em relação à insatisfação corporal, os meninos pré-puberes e púberes apresentaram maior probabilidade desse sentimento do que os atletas pós-púberes (FORTES, ALMEIDA, FERREIRA, 2012b). Entretanto, vale ressaltar que ambos os estudos avaliaram diversas modalidades esportivas em conjunto. Ainda existe a necessidade de estudar o processo maturacional em alguns esportes específicos.

Os indicadores antropométricos (percentual de gordura $[\% \mathrm{G}]$ e índice de massa corporal [IMC]) também foram avaliados como possíveis fatores influenciadores da insatisfação corporal e dos comportamentos de risco para transtornos alimentares (FORTES, 
ALMEIDA, FERREIRA, 2012a; FORTES, ALMEIDA, FERREIRA, 2013b). Ambas as investigações encontraram que o percentual de gordura foi a única variável que influenciou a insatisfação corporal em meninas e meninos e que os comportamentos de risco para transtornos alimentares em jovens atletas parecem não ser influenciados por características antropométricas (FORTES, ALMEIDA, FERREIRA, 2012a; FORTES, ALMEIDA, FERREIRA, 2013b). A avaliação do \%G e do IMC tem sido utilizados, não só pela possível influência destes a fatores psicológicos, mas também como ferramenta de caracterização da amostra (PERINI et al., 2009).

A influência de fatores afetivos, antropométricos e sociodemográficos sobre os comportamentos de risco para transtornos alimentares em meninas atletas foram analisadas por Fortes, Oliveira e Ferreira (2012). Os autores não identificaram diferença significativa da etnia, dos níveis econômico e competitivo sobre as pontuações do EAT-26. Apenas o \%G e a insatisfação corporal exerceram influência significativa sobre os comportamentos de risco para transtornos alimentares de atletas femininas.

Dessa forma, ressalta-se que, nacionalmente, é presente a tentativa de associar um dos componentes da imagem corporal - insatisfação corporal - e os comportamentos de risco para transtornos alimentares a diversas variáveis. Internacionalmente, este padrão também foi identificado.

\section{As pesquisas internacionais sobre imagem corporal e comportamentos de risco para transtornos alimentares}

Inicialmente, quanto ao tipo de metodologia empregada, apenas um estudo essencialmente qualitativo foi identificado (FRANCISCO; ALARCÃO; NARCISO, 2012), o qual buscou investigar as características específicas do ambiente da dança e ginástica que poderiam torná-los fatores de risco para desenvolvimento de transtornos alimentares. Os autores identificaram que as pressões para a magreza e restrição alimentar como caminho para atingir o ideal corporal são comuns a ginastas e bailarinos. Entretanto, os bailarinos percebem o ambiente de treino como um sistema específico com regras e cultura própria mais do que as ginastas.

Do total de estudos encontrados, quatro utilizaram métodos de pesquisa mistos quali-quantitativos (MARTINSEN, SUNDGOT-BORGEN, 2013; THIEL et al., 2011; TORSTVEIT, ROSENVINGE, SUNDGOT-BORGEN, 2008; TORSTVEIT, SUNDGOTBORGEN, 2012). Nesses estudos, inicialmente foi utilizada uma ferramenta quantitativa para rastreio de comportamentos de risco para transtornos alimentares na população em questão. Em seguida, foi realizada uma entrevista clínica com aqueles indivíduos que se destacaram da população em geral. Vale ressaltar que os estudos de Martinsen e Sundgot-Borgen (2013) e Torstveit, Rosenvinge e Sundgot-Borgen (2008) buscaram identificar a prevalência de comportamentos de risco para transtornos alimentares em adolescentes atletas e não atletas. $\mathrm{O}$ primeiro estudo encontrou maior prevalência desses comportamentos em atletas do que em adolescentes controle, e maior em meninas quando comparadas ao sexo oposto. Já o segundo identificou maior número de atletas de esportes estéticos $(46,7 \%)$ com sintomas clínicos para transtornos alimentares do que de esportes não estéticos $(19,8 \%)$ e controle $(21,4 \%)$. Esses achados indicam que, a população de meninas adolescentes praticantes de esportes estéticos, merece atenção por parte dos profissionais envolvidos em sua prática esportiva, devido à maior propensão a desencadearem transtornos alimentares. 
Quanto ao delineamento, foram identificados apenas três estudos longitudinais (BUCHHOLZ et al., 2008; KRENTZ, WARSCHBURGER, 2013; MARTINSEN et al., 2014). Krentz e Warschburger (2013) examinaram fatores de risco para transtornos alimentares relacionados ao esporte em dois momentos (inicial e um ano depois). Os autores demonstraram que a busca pela magreza como forma de melhorar o desempenho esportivo é um fator preditivo para o desenvolvimento de transtornos alimentares em atletas. Dessa forma, os atletas estão em maior risco para o desenvolvimento de transtornos alimentares se eles acreditarem que é possível melhorar a performance com o controle de peso. Por outro lado, Buchholz et al. (2008) e Martinsen et al. (2014) avaliaram a efetividade de um programa de prevenção para transtornos alimentares em atletas. Ambos os estudos apontaram melhora nos comportamentos após a intervenção. Assim, são incentivados programas multidisciplinares que proporcionem às atletas melhores condições psicológicas no ambiente do esporte de alto rendimento.

Em relação às ferramentas de avaliação, foi identificada uma variedade de instrumentos utilizados nos artigos internacionais. Foram aplicados tanto questionários padronizados, quanto com questões próprias, desenvolvidas pelos autores dos estudos. Para a avaliação da imagem corporal, alguns estudos se valeram de escalas de silhuetas (FRANCISCO, NARCISO, ALARCÃO, 2013; KRENTZ, WARSCHBURGER, 2013; KRENTZ, WARSCHBURGER, 2011; ROSENDAHL et al., 2009), enquanto que apenas um estudo utilizou o Body Shape Questionnaire (ANDERSON; PETRIE; NEUMANN, 2011). Para ilustrar, Rosendahl et al. (2009) encontraram que 70,3\% das meninas e 59,3\% dos meninos consideram não possuir o corpo ideal, sendo que a maioria das meninas desejam diminuir a silhueta, enquanto os meninos, aumentar. Vale ressaltar ainda que, na maioria das vezes, o objetivo principal dos estudos identificados não era a avaliação da insatisfação corporal, mas sim sua relação com outras variáveis, ou até mesmo sua utilização como covariável nas análises estatísticas. Dessa forma, os dados referentes à prevalência de atletas insatisfeitos com o corpo não foram apresentados nos artigos.

Para a avaliação de comportamentos de risco para transtornos alimentares, o Eating Attitudes Test-26 e o Eating Disorders Inventory estão entre os instrumentos mais utilizados. Destaca-se que alguns estudos encontrados objetivaram avaliar a prevalência de comportamentos de risco para transtornos alimentares. Os resultados apontados pelos pesquisadores apresentam grande variação quanto a porcentagem de atletas em condição de risco, variando desde em $1,9 \%$ dos bailarinos diagnosticados clinicamente para transtornos alimentares (HERBRICH et al., 2011) a 44,7\% de atletas com comportamentos de risco para transtornos alimentares (MARTINSEN; SUNDGOT-BORGEN, 2013). Essa grande variação pode ter ocorrido devido à utilização de instrumentos avaliativos diferentes, ou mesmo pela sensibilidade dos instrumentos utilizados.

De maneira similar ao encontrado nacionalmente, na maioria dos estudos internacionais, diferentes modalidades esportivas foram consideradas em conjunto. Em contrapartida, poucas foram as pesquisas que avaliaram algum esporte de maneira específica, entre eles, destacaram-se as modalidades: ballet $(\mathrm{n}=6$ estudos), patinação artística $(\mathrm{n}=4$ estudos), ginástica rítmica ( $\mathrm{n}=2$ estudos) e ginástica artística $(\mathrm{n}=2$ estudos).

Em relação a estudos que avaliaram outras variáveis, a motivação (SCOFFIER; CORRION; D'ARRIPE-LONGUEVILLE, 2013) e a influência de pais (FRANCISCO; NARCISO; ALARCÃO, 2013) foram associadas ao comportamento alimentar, sendo que ambas foram consideradas fatores de risco, apontando a necessidade de avaliação nesse 
público. Destaca-se ainda a tentativa de relacionar o perfeccionismo à imagem corporal (DUNN; CRAFT; DUNN, 2011) e aos comportamentos de risco para transtornos alimentares (GOMES; SILVA, 2010). Segundo esses estudos, parece que o perfeccionismo é uma variável que está relacionada tanto com a imagem corporal quanto com os comportamentos de risco para transtornos alimentares em atletas.

Por fim, salienta-se que seis artigos encontrados buscaram testar modelos teóricos. Dentre eles destaca-se o estudo realizado por Anderson, Petrie e Neumann (2011) que objetivou verificar a validade de um modelo para sintomas bulímicos em duas amostras independentes: atletas colegiais de natação e ginástica. Após a confirmação do modelo de influência, os autores concluíram que as pressões socioculturais gerais são influentes, mas as pressões do ambiente esportivo em relação à manutenção do peso corporal e da aparência física são ainda mais presentes e negativas para atletas do sexo feminino. Isso demonstra a existência de um modelo teórico específico para atletas relacionado aos comportamentos de risco para transtornos alimentares. Entretanto, pesquisas com essa característica não foram identificadas na literatura brasileira. E, dessa forma, ainda são incentivados estudos que busquem confirmar a validade desses modelos no Brasil.

\section{Considerações Finais}

A partir das discussões acima levantadas, pode-se concluir que o Brasil, no cenário mundial, é o país que apresenta o maior número de publicações considerando modalidades esportivas estéticas em conjuntos a outros esportes. Os resultados dos estudos nacionais apontaram prevalências semelhantes de insatisfação corporal e comportamentos de risco para transtornos alimentares em atletas. Isso pode ter ocorrido devido à utilização dos mesmos instrumentos de medida: o Body Shape Questionnaire e o Eating Attitudes Test-26. Apesar de não terem sido criados especificamente para atletas, estes ainda são as ferramentas mais utilizadas no Brasil para avaliação desse público.

Internacionalmente, as metodologias utilizadas pelos estudos foram mais diversificadas (alguns estudos qualitativos, de métodos mistos e longitudinais foram encontrados), assim como a variedade de instrumentos avaliativos. Isso teve repercussão em relação aos achados dos estudos quanto às prevalências de insatisfação corporal e comportamentos de risco para transtornos alimentares, nos quais foi possível observar resultados mais variantes. Também foram avaliados modelos teóricos para melhor entendimento do processo de desencadeamento dos transtornos alimentares em atletas.

Em relação à amostra dos estudos analisados, três considerações devem ser feitas. Inicialmente, quanto ao tamanho amostral, os estudos internacionais apresentaram grande diversidade desse número variando de 22 (FRANCISCO; ALARCÃO; NARCISO, 2012) a 2067 atletas (SCHAAL et al., 2011). Já entre os estudos nacionais, o tamanho amostral variou de 47 (FORTES et al., 2013) a 580 (8 dos 16 estudos nacionais). Possivelmente, dois motivos que levaram a essa grande disparidade são: o próprio desenho do estudo (estudos qualitativos geralmente têm amostras menores quanto comparados a estudos quantitativos, e estudos transversais têm maiores amostras do que estudos longitudinais); e a inclusão de modalidades em específico ou em conjunto (estudos que englobam diversos esportes tendem a ter amostras maiores).

Em segundo lugar, é necessário destacar a característica das amostras dos estudos encontrados quanto ao sexo. Não foram identificados estudos que considerassem modalidades 
esportivas estéticas cuja amostra se constituísse apenas de indivíduos do sexo masculino. Ressalta-se que este fato possivelmente ocorreu pela própria característica dessas modalidades que, em geral, abarcam um número maior de meninas do que meninos. O terceiro ponto a ser considerado com relação à amostra diz respeito à utilização ou não de grupo controle. Vale frisar que a utilização do grupo controle é essencial nos estudos experimentais, nos quais se busca a verificação de relação de causa e efeito entre as variáveis. Dessa forma, destaca-se a necessidade de pesquisas que considerem essa característica.

Com relação às áreas de estudo/departamentos de onde provêm os artigos analisados no Brasil e internacionalmente, a grande maioria é proveniente da área da Educação Física, seguido pela Psicologia. Dessa forma, entende-se que apesar do estudo da imagem corporal e dos comportamentos de risco para transtornos alimentares em atletas ser considerado como um assunto de interesse de ambas as áreas, o mesmo têm sido investigado, predominantemente, por pesquisadores da Educação Física. Essa temática é relevante com impacto importante tanto no âmbito da saúde física e mental quanto no âmbito educativo e esportivo.

Como limitação do presente estudo, destaca-se, inicialmente, a verificação da produção científica relativa a artigos publicados em periódicos, não envolvendo buscas em bases de dissertações e teses e anais de congressos. Além disso, a escolha do corte temporal a partir de 2008 impossibilitou a análise da evolução absoluta das publicações. Contudo, optouse por essas escolhas, pois os artigos científicos apresentam informações completas e diretas, bem como para analisar os artigos mais atualizados sobre o tema.

São incentivados estudos brasileiros que utilizem métodos longitudinais e com grupo controle, possibilitando o estabelecimento de relação de causa e efeito entre as variáveis. Além disso, ainda existe carência de estudos que desenvolvam instrumentos específicos para a avaliação da imagem corporal e dos comportamentos de risco para transtornos alimentares de atletas, possibilitando inclusive a avaliação das especificidades de alguns esportes, especialmente os esportes estéticos.

\title{
BODY DISSATISFACTION AND EATING BEHAVIOR IN ATHLETES FROM AESTHETIC SPORTS: A REVIEW
}

\begin{abstract}
The aim of this study was to analyze the scientific literature about body dissatisfaction and risk behaviors for eating disorders in adolescent athletes of aesthetic sports. We analyzed 45 studies published between the years of 2008 and 2015. In Brazil, a similar prevalence of body dissatisfaction and risky behavior for eating disorders in athletes were identified, possibly by the use of the same assessment instruments. Internationally, a greater variety of assessment instruments was identified, which was reflected in differences in prevalence of these behaviors. Brazilian studies using longitudinal methods and control group are encouraged.
\end{abstract}

Keywords: Body Image. Feeding Behavior. Eating Disorders. Adolescent. 


\section{INSATISFACCIÓN CORPORAL Y COMPORTAMIENTO ALIMENTAR EN ATLETAS DE DEPORTES ESTÉTICOS: UNA REVISIÓN}

\section{Resumen}

El objetivo fue analizar la producción científica sobre la insatisfacción corporal y conductas de riesgo para los trastornos de la alimentación en los atletas adolescentes de los deportes estéticos. Se analizaron 45 estudios publicados entre 2008 y 2015. En Brasil, fueron identificadas prevalencias similares de insatisfacción corporal y los comportamientos de riesgo para el trastorno de la alimentación en los atletas, posiblemente mediante el uso de las mismas herramientas de evaluación. A nivel internacional, se identificó una mayor variedad de instrumentos de evaluación, que reflejan las diferencias en la prevalencia de estas conductas. Se anima a los estudios brasileños utilizando métodos longitudinales y grupo de control.

Palabras clave: Imagen Corporal. Conducta Alimentar. Trastornos de alimentación. Adolescente.

\section{Referências}

AMERICAN PSYCHIATRIC ASSOCIATION. Diagnostic and statistical manual of mental disorders. 5. ed. Washington, DC: American Psychiatric Association, 2014.

ANDERSON, C. M.; PETRIE, T. A.; NEUMANN, C. S. Psychosocial correlates of bulimic symptoms among NCAA division-I female collegiate gymnasts and swimmers/divers. Journal of Sport \& Exercise Psychology, Stirling, v. 33, n. 4, p. 483-505. 2011.

BUCHHOLZ, A. et al. BodySense: An evaluation of positive body image intervention on sport climate for female athletes. Eating Disorders, Charleston, v. 16, n. 4, 2008. p. 308-321.

CONTI, M. A. Os aspectos que compõem o conceito de imagem corporal pela ótica do adolescente. Revista Brasileira de Crescimento e Desenvolvimento Humano, São Paulo, v. 18, n. 3, 2008. p. 240-253.

DUNN, J. G. H.; CRAFT, J. M.; DUNN, J. C. Comparing a domain-specific and global measure of perfectionism in competitive female figure skaters. Journal of Sport Behavior, Alabama, v. 34, n. 1, 2011. p. 25-46.

FERREIRA, M. E. C.; CASTRO, M. R.; MORGADO, F. F. R. Imagem corporal: Reflexões, diretrizes e práticas de pesquisa. Juiz de Fora: Editora UFJF, 2014.

FORTES, L. S.; ALMEIDA, S. S.; FERREIRA, M. E. C. Influência da ansiedade nos comportamentos de risco para os transtornos alimentares em ginastas. Revista Brasileira de Atividade Física e Saúde, Pelotas, v. 18, n. 5, 2013a. p. 546-553.

Indicadores antropométricos de insatisfação corporal e de comportamentos alimentares inadequados em jovens atletas. Revista Brasileira de Medicina do Esporte, Niterói, v. 19, n. 1, 2013b. p. 35-39.

Imagem corporal no contexto esportivo. In: FERREIRA, M. E. C.; CASTRO, M. 
R.; MORGADO, F. F. R. Imagem corporal: Reflexões, diretrizes e práticas de pesquisa. Juiz de Fora: Editora UFJF, 2014a. p. 279-306.

A internalização do ideal de magreza afeta os comportamentos alimentares inadequados em atletas do sexo feminino da ginástica artística?. Revista da Educação Física/UEM, Maringá, v. 25, n. 2, 2014b. p. 181-191.

Impacto de variáveis antropométricas sobre a insatisfação corporal e o comportamento alimentar em jovens atletas. Jornal Brasileiro de Psiquiatria, Rio de Janeiro, v. 61, n. 4, 2012a. p. 235-241.

Processo maturacional, insatisfação corporal e comportamento alimentar inadequado em jovens atletas. Revista de Nutrição, Campinas, v. 25, n. 5, 2012b. p. 575-586.

Relação entre comportamentos de risco para transtornos alimentares e processo maturacional em jovens atletas. Revista Brasileira de Atividade Física e Saúde, Pelotas, v. 17, n. 5, 2012. p. 379-395.

Comparação da insatisfação corporal e do comportamento alimentar inadequado em atletas adolescentes de diferentes modalidades esportivas. Revista Brasileira de Educação Física e Esporte, São Paulo, v. 25, n. 4, 2011. p. 707-716.

Comportamento alimentar inadequado: comparações em função do comprometimento ao exercício. Arquivos Brasileiros de Psicologia, Rio de Janeiro, v. 65, n. 2, 2013. p. 230-242.

FORTES, L. S.; KAKESHITA, I. S. et al. Eating behaviours in youths: A comparison between female and male athletes and non-athletes. Scandinavian Journal of Medicine \& Science in Sports, Berlin, v. 24, n. 1, 2013. p. e62-e68.

FORTES, L. S.; NEVES, C. M. et al. Insatisfação corporal, comprometimento psicológico ao exercício e comportamento alimentar em jovens atletas de esportes estéticos. Revista Brasileira de Cineantropometria \& Desempenho Humano, Florianópolis, v. 15, n. 6, 2013. p. 695-704.

FORTES, L. S.; OLIVEIRA, F. G.; FERREIRA, M. E. C. Influência de fatores afetivos, antropométricos e sociodemográficos sobre o comportamento alimentar em jovens atletas. Jornal Brasileiro de Psiquiatria, Rio de Janeiro, v. 61, n. 3, 2012.

p. 148-153.

FRANCISCO, R.; ALARCÃO, M.; NARCISO, I. Aesthetic sports as high-risk contexts for eating disorders: Young elite dancers and gymnasts perspectives. The Spanish Journal of Psychology, Madrid, v. 15, n. 1, 2012. p. 265-274.

FRANCISCO, R.; NARCISO, I.; ALARCÃO, M. Parental Influences on Elite Aesthetic 
Athletes' Body Image Dissatisfaction and Disordered Eating. Journal of Child and Family Studies, New York, v. 22, n. 8, 2013. p. 1082-1091.

GOMES, R.; SILVA, L. Desordens alimentares e perfeccionismo: Um estudo com atletas portugueses. Psicologia em Revista, Belo Horizonte, v. 16, n. 3, 2010. p. 469-489.

HERBRICH, L. et al. Anorexia athletica in pre-professional ballet dancers. Journal of Sports Sciences, v. 29, n. 11, 2011. p. 1115-1123.

KLINKOWSKI, N. et al. Psychopathology in elite rhythmic gymnasts and anorexia nervosa patients. European Child \& Adolescent Psychiatry, v. 17, n. 2, 2008. p. 108-113.

KRENTZ, E. M.; WARSCHBURGER, P. A longitudinal investigation of sports related risk factors for disordered eating in aesthetic sports. Scandinavian Journal of Medicine \& Science Sports, Berlin, v. 23, n. 3, 2013. p. 303-310.

KRENTZ, E. M.; WARSCHBURGER, P. Sports-related correlates of disordered eating in aesthetic sports. Psychology of Sport and Exercise, London, v. 12, n. 4, 2011. p. 375-382.

MALINA, R. M. et al. Role of intensive training in the growth and maturation of artistic gymnasts. Sports Medicine, v. 43, n 9, 2013. p. 783-802.

MARTINSEN, M. et al. Preventing Eating Disorders among Young Elite Athletes: A Randomized Controlled Trial. Medicine \& Science in Sports \& Exercise, New York, v. 46, n. 3, 2014. p. 435-447.

MARTINSEN, M.; SUNDGOT-BORGEN, J. Higher prevalence of eating disorders among adolescent elite athletes than controls. Medicine \& Science in Sports \& Exercise, New York, v. 45, n. 6, 2013. p. 1188-1197.

NEVES, C. M. et al. Comportamentos alimentares em ginastas de elite: associação com o perfeccionismo e o estado de humor. Revista da Educação Física/UEM, Maringá, v. 24, n. 3, 2013. p. 359-369.

ONIS, M. et al. Development of a WHO growth reference for school-aged children and adolescents. Bulletin of the World Health Organization, v. 85, n. 9, 2007. p. 660-667.

PERINI, T. A. et al. Transtorno do comportamento alimentar em atletas de elite de nado sincronizado. Revista Brasileira de Medicina do Esporte, Niterói, v. 15, n. 1, 2009. p. 5457.

PETRIE, T. A.; GREENLEAF, C. Body image and athleticism. IN: CASH, T.; SMOLAK, L. Body image: a handbook of science, practice, and prevention. 2. ed. (pp. 67-75). New York: The Guilford Press, 2011.

ROSENDAHL, J. et al. Dieting and disordered eating in German high school athletes and 
non-athletes. Scandinavian Journal of Medicine \& Science Sports, Berlim, 19(5), 2009. p. 731-739.

SCHAAL, K. et al. Psychological balance in high level athletes: Gender based differences and sport-specific patterns. PlosOne, v. 6, n. 5, 2011. p. 1-9.

SCOFFIER, S.; CORRION, K.; D'ARRIPE-LONGUEVILLE, F. Effects of achievement goals on female aesthetic athletes' disordered eating attitudes. Science \& Sports, v. 28, n. 6, 2013. p. e151-e157.

STREINER, D. L. Starting at the beginning: An introduction to coefficient alpha and internal consistency. Journal of Personality Assessment, v. 80, n. 1, 2003. p. 99-103.

THIEL, A. et al. The German young olimpic athletes' lifestyle and health management study (GOAL Study): Desing of a mixed-method study. BMC Public Health, v. 11, n. 31, 2011. p. 410-420.

TORSTVEIT, M. K.; ROSENVINGE, J. H.; SUNDGOT-BORGEN, J. Prevalence of eating disorders and the predictive power of risk models in female elite athletes: A controlled study. Scandinavian Journal of Medicine \& Science Sports, Berlin, v. 18, n. 1, 2008. p. 108-118.

TORTVEIT, M. K., SUNDGOT-BORGEN, J. Are under- and overweight female elite athletes thin and fat? A controlled study. Medicine and Science Sports and Exercise, New York, v. 44, n. 5 , 2012. p. $949-957$.

VIEIRA, J. L. L. et al. Distúrbios de atitudes alimentares e sua relação com o crescimento físico de atletas paranaenses de ginástica rítmica. Motriz, Rio Claro, v.15, n.3, 2009. p.552561.

Recebido em: 26/08/2015

Revisado em: 15/09/2015

Aprovado em: 29/09/2015

Endereço para correspondência:

eujuly90@hotmail.com

Juliana Fernandes Filgueiras Meireles

Universidade Federal de Juiz de Fora, Faculdade de Educação Física.

Campus universitário

Cidade universitária

36036-330 - Juiz de Fora, MG - Brasil 